\title{
A Randomized, Double-Blind, Placebo-Controlled, Clinical Trial of High-Dose, Short-Term Vitamin D Administration in the Prevention of Acute Kidney Injury after Cardiac Surgery
}

\author{
Pegah Eslami ${ }^{a}$ Manouchehr Hekmat ${ }^{b}$ Mahmoud Beheshti ${ }^{b} \quad$ Ramin Baghaei $^{\mathrm{b}}$ \\ Seyed Mohsen Mirhosseini ${ }^{b}$ Fatemeh Pourmotahari ${ }^{b}$ Seyed Ali Ziai ${ }^{c}$ \\ Mahnoosh Foroughi ${ }^{\mathrm{a}}$ \\ ${ }^{a}$ Cardiovascular Research Center, Shahid Beheshti University of Medical Sciences, Tehran, Iran; ${ }^{b}$ Clinical Research \\ and Development Center, Shahid Modarres Hospital, Shahid Beheshti University of Medical Sciences, Tehran, Iran; \\ 'Department of Pharmacology, School of Medicine, Shahid Beheshti University of Medical Sciences, Tehran, Iran
}

\section{Keywords}

Acute renal failure $\cdot$ Cardiac surgery $\cdot$ Inflammation .

Biomarkers · Nutrition

\begin{abstract}
Background: Acute kidney injury (AKI) after cardiac surgery is a relatively common complication affecting short- and long-term survival. The renoprotective effect of vitamin $D$ (VitD) has been confirmed in several experimental models. This study was conducted to evaluate the effect of high-dose VitD administration in patients with VitD insufficiency on the incidence of postoperative AKI, the urinary level of tubular biomarkers, and serum anti-inflammatory biomarker after coronary artery bypass graft. Design and Method: In this randomized double-blind controlled clinical trial, the patients were randomly allocated to either the VitD group $(n=50)$, receiving 150,000 IU VitD tablets daily for 3 consecutive days before surgery or the control group $(n=61)$, receiving placebo tablets. Results: There was no difference in the incidence of postoperative AKI between the groups. Both of the urinary levels of interleukin-18 and kidney injury molecule-1 were significantly increased after the operation $(p<0.001$,
\end{abstract}

for both). Also, the serum level of interleukin-10 was increased after 3 days of VitD supplementation ( $p=0.001)$. In comparison with the control group, it remained on a higher level after the operation $(p<0.001)$ and the next day $(p=$ 0.03 ). The patients with AKI had more postoperative bleeding and received more blood transfusion. Conclusion: VitD pretreatment was unable to impose any changes in the incidence of AKI and the urinary level of renal biomarkers. However, high-dose administration of VitD may improve the antiinflammatory state before and after the operation. Further studies are needed to assess the renoprotective effect of VitD on coronary surgery patients.

(c) 2021 S. Karger AG, Basel

\section{Introduction}

Cardiac surgery associated-acute kidney injury (CSAAKI) is a known complication affecting up to $30 \%$ of the patients after cardiac surgery [1-4]. Despite the advances in perioperative care, due to the aging population and in association with several comorbidities, the incidence and mortality of CSA-AKI have not been decreased during karger@karger.com

(c) 2021 S. Karger AG, Base

www.karger.com/crm

Karger ${ }^{\prime}=$
Mahnoosh Foroughi

Cardiovascular Research Center, Modarres Hospital

Shahid Beheshti University of Medical Sciences

Saadat Abad, Tehran 1998734383 (Iran)

m_foroughi@sbmu.ac.ir 
this decade, and it is associated with high incidence of adverse outcomes even after complete renal recovery [1-3].

During cardiac surgery with cardiopulmonary bypass (CPB), the stimulation of inflammatory cascade and oxidative stress, severe hemodynamic disturbance with renal hypoperfusion, and using nephrotoxic agents are the well-defined risk factors for CSA-AKI development [1, $3]$. The nonphysiologic state of CPB activates the local and systemic inflammatory response, ischemia-reperfusion injury, and oxidative stress affecting pathologic changes on all the organs including kidney [1]. Recruitments of inflammatory cells in renal parenchymal increase the tubular damage markers, and the disturbance in the autoregulation mechanism of perfusion provides a complex interaction between vascular endothelial dysfunction and tubular epithelial damage, observed in patients with CSA-AKI $[1,5]$. The preventive strategies, such as corticosteroids, ultrafiltration, and minimal extracorporeal circulation used during $\mathrm{CPB}$, failed to reduce AKI $[1,2,6,7]$.

In addition to calcium homeostasis, vitamin D (VitD) has a pleiotropic effect on the immune system. A growing body of evidence suggests its anti-inflammatory, antiapoptotic, and antioxidative properties [8-13]. VitD treatment has been found to attenuate renal injury by inhibiting mitochondrial dysfunction and reduce apoptosis in the models of ischemic reperfusion induced by AKI [14-17]. The proximal tubular cells have been the main target of VitD against the infiltration of inflammatory cells in the experimental nephropathies model $[12,14-$ 17]. In clinical studies, the attenuation of inflammatory state and renal injury through VitD approach has not been evaluated yet. This study was conducted to determine the renoprotective effect of $\mathrm{VitD}$ pretreatment on postoperative CSA-AKI incidence, urinary level of proximal tubular biomarkers of the kidney, and serum antiinflammatory interleukin-10 (IL-10) level in patients with cardiac surgery.

\section{Methods}

\section{Study Design}

The protocol of this randomized double-blind placebo-controlled study was approved by the Ethics Committee of the University, and it was registered on https://www.irct.ir (ID: IRCT20180131038578N1). The inclusion criteria were as follows: patients referred for first time elective and isolated coronary artery bypass graft (CABG) by using $\mathrm{CPB}$, who had VitD insufficiency $(<20 \mathrm{ng} / \mathrm{mL})$ as described in the previous studies, and with normal kidney function $(\mathrm{Cr}<1.5 \mathrm{mg} / \mathrm{dL})[9,11]$. The exclusion criteria were as follows: a history of treatment with VitD, recent myocardial infarction, urgent $\mathrm{CABG}$, nonisolated coronary surgery, a combination of coronary artery disease and other cardiac pathologies, the presence of acute or chronic inflammatory diseases, and using nephrotoxic drug or contrast media 2 weeks before the operation. By exclusion of well-known risk factors as confounding factors, an effort has been made to evaluate the CPB effect on kidney function in a relative homogenous population (only CABG patients).

\section{Interventions}

After obtaining written informed consent, patients were randomly allocated (using a computer-generated random number) to either receive 50,000 units of VitD tablet 3 times a day, for 3 days before the operation (VitD group, $n=50$ ) or placebo, inactive medication tablet (control group, $n=61$ ) at the same period time (Fig. 1). For both groups, the coronary artery bypass was performed in the culprit lesions by a surgical team. All patients were treated according to a fixed protocol for general anesthesia, surgical, hemodynamic, and CPB management, which has already been described in detail [18]. Mean arterial pressure was maintained on 60-80 $\mathrm{mm} \mathrm{Hg}$, and deviations were corrected with nitroglycerin and phenylephrine (after optimization of preload, CVP $\geq 10 \mathrm{~cm}$ $\mathrm{H}_{2} \mathrm{O}$ ). Hemoglobin concentrations $<7 \mathrm{~g} / \mathrm{dL}$ during $\mathrm{CPB}$ and $<8 \mathrm{~g} /$ $\mathrm{dL}$ after $\mathrm{CPB}$ and in the ICU were used, as transfusion thresholds. Investigators, patients, and the medical team were blinded to the treatment allocation.

\section{Sample Collection and Biochemical Assays}

The urinary sample for measuring kidney injury molecule-1 (KIM-1) and interleukin-18 (IL-18) and blood sample for measuring serum level of IL-10 were collected at baseline (T1), just before the operation (after drug intervention) (T2), at the end of the operation (T3), and the day after the operation (T4). The urinary KIM-1 level was assessed using a commercial ELISA test kit (BioAssay Works, LLC, Ijamsville, MD, USA). Moreover, the urinary IL-18 level was measured by ELISA test kit (IBL International $\mathrm{GmbH}$, Hamburg; Germany). In addition, serum IL-10 level was measured by ELISA test kit (IBL International GmbH, Hamburg; Germany). VitD was measured by an immunoluminometric assay. The samples were centrifuged at 2,500 rpm for $15 \mathrm{~min}$ within $1 \mathrm{~h}$ after sampling, and they were stored at $-20^{\circ} \mathrm{C}$ until analysis. These tests were performed according to manufactures instructions in a blinded fashion.

\section{Outcomes}

The primary outcome was the AKI development through daily serum Cr measurement between the groups after CABG. According to the AKI Network (KDIGO) criteria, AKI was described as an increase in postoperative serum $\mathrm{Cr} \geq 0.3 \mathrm{mg} / \mathrm{dL}$ from the baseline within $48 \mathrm{~h}$ after CABG $[1,3]$. The secondary outcomes were the degree of change in inflammatory renal biomarkers by measuring the urinary IL-18 and KIM-1 and the serum level of anti-inflammatory IL-10.

\section{Statistical Analysis}

Continuous and categorical variables were presented as mean \pm standard deviation and percentages, respectively. Repeated measures of the ANOVA and multiple comparisons were used to determine the differences at specific time points, followed by a
Vitamin D and Renal Inflammatory

Response during Cardiac Surgery
Cardiorenal Med 2021;11:52-58

DOI: $10.1159 / 000511058$ 


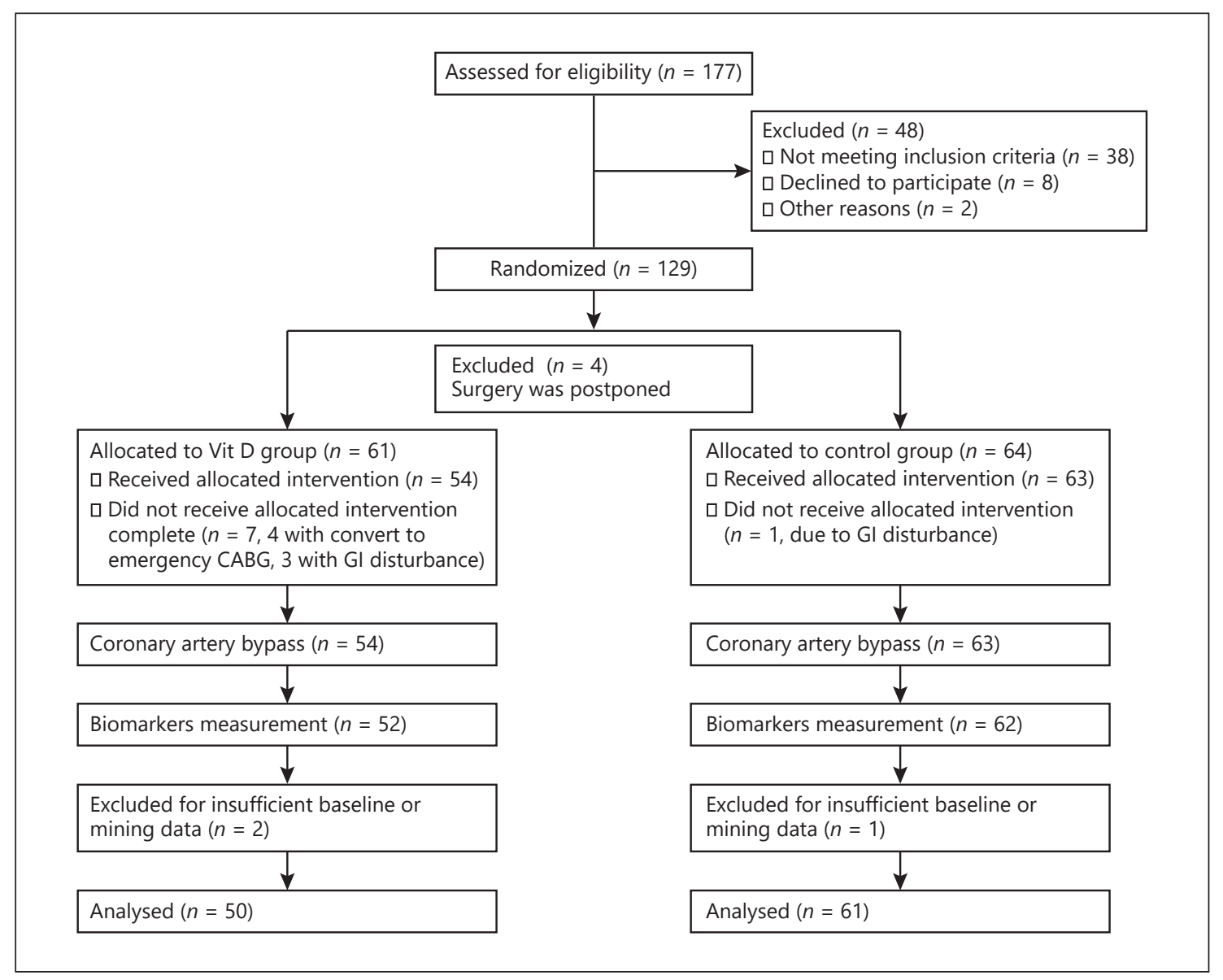

Fig. 1. Study flow chart.

Tukey's post hoc test in case of significant difference between groups. Type I error was corrected by the Bonferroni method. Furthermore, the Kolmogorov-Smirnov test was performed for the normal distribution of data. Continuous and categorical variables between groups were compared using Student's $t$ test (or MannWhitney test for unequal variance) and $\chi^{2}$, respectively. All statistical analyses were performed using STATA software, version 13 (StataCorp. College Station, TX, USA), and $p$ value $<0.05$ was considered to be significant.

\section{Results}

The study population consisted of 111 patients with CABG enrolled between February 2019 and August 2019 in a tertiary university hospital (Fig. 1). The demographic and perioperative characteristics of patients were comparable between groups (Table 1). There was no difference in underlying medical conditions (diabetes, hypertension, and hyperlipidemia) and medication between the groups. Also, there was no difference in perioperative management, duration of surgery (aortic clamp time and pump time), urine output and fluid intake during operation, administration of blood products, and vasoactive drugs between the groups.

In addition, there was no statistically significant difference in the serum $\mathrm{Cr}$ level in the first 3 days after the operation between both groups $(p=0.5)$. Furthermore, there was no significant difference in the incidence of postoperative AKI between the groups (12\% in VitD vs. $16 \%$ in the control group, $p=0.1$ ). Additionally, none of the patients needed renal replacement therapy.

The level of urinary IL-18 and KIM- 1 at the baseline was similar in both groups. The urinary level of IL-18 and KIM-1 was significantly increased after ICU arrival and the first day after the operation, with no significant difference between groups ( $p=0.6$ and $p=0.7$, respectively) (Fig. 2). The serum level of IL-10 was significantly increased in VitD group after intervention in all periods 
Table 1. Demographic, intraoperative, and postoperative data

\begin{tabular}{lccl}
\hline & VitD group, & Control group, \\
$n=50$ & $p$ & $p$ \\
& & & \\
& & & \\
& $58 \pm 7$ & $58 \pm 9$ & 0.6 \\
Age, years & $42(84 \%)$ & $47(77 \%)$ & 0.2 \\
Sex $(\mathrm{m})$ & $12 \pm 6$ & $11 \pm 5$ & 0.3 \\
VitD, ng/mL & $15 \pm 8$ & $14 \pm 8$ & 0.5 \\
ESR, mm/h & $47 \pm 10$ & $48 \pm 9$ & 0.4 \\
EF, $\%$ & & & \\
Risk factors, $n$ (\%) & $12(24)$ & $16(26)$ & 0.4 \\
FH & $28(56)$ & $32(52)$ & 0.4 \\
SM & $8(16)$ & $4(6)$ & 0.08 \\
PCI & $4(8)$ & $2(3)$ & 0.2 \\
CVA & $22(44)$ & $26(42)$ & 0.5 \\
MI & $20(40)$ & $36(59)$ & 0.06 \\
DM & $16(32)$ & $25(40)$ & 0.1 \\
HLP & $30(60)$ & $38(62)$ & 0.4 \\
HTN & & & \\
Perioperative data & $54 \pm 7$ & $56 \pm 12$ & 0.2 \\
Clamp time, min & $102 \pm 14$ & $102 \pm 20$ & 0.9 \\
Pump time, min & $3.6 \pm 0.4$ & $3.5 \pm 0.5$ & 0.4 \\
Graft NO & $2(4 \%)$ & 0 & 0.1 \\
IABP & $1.1 \pm 1.1$ & $1.4 \pm 1.3$ & 0.2 \\
PCOR, unit & $6.8 \pm 2.5$ & $8.6 \pm 4.6$ & 0.02 \\
Ventilation, h & $0.5 \pm 0.7$ & $0.8 \pm 0.9$ & 0.1 \\
PCICU, unit & $382 \pm 250$ & $395 \pm 252$ & 0.7 \\
Bleeding, mL & $6(12)$ & $10(16)$ & 0.1 \\
AKI, $n$ (\%) & & \\
\hline
\end{tabular}

The common patients' related risk factors for AKI (DM, HTN, aging, and heart contractility status) were similar between groups. By choosing only CABG patients, the effect of procedure-related factors as the possible confounding variable was excluded. The common causes of postoperative AKI (cardiogenic shock and need to IABP, need to blood transfusion and excessive blood loss through chest tubes) were similar between groups. Data are reported as numbers (\%) for categorical variables and as mean \pm standard deviation for continuous variables. ESR, erythrocyte sedimentation rate; $\mathrm{FH}$, family history; SM, smoking history; $\mathrm{PCI}$, percutaneous coronary intervention; CVA, cerebrovascular accident; MI, myocardial infarction; DM, diabetes mellitus; HLP, hyperlipidemia; HTN, hypertension; IABP, intra-aortic balloon pump; PCOR, packed cell used in operating room; PCICU, packed cell used in ICU; AKI, acute kidney injury; VitD, vitamin D; CABG, coronary artery bypass graft.

$(p<0.001)$. The level of IL-10 at baseline was comparable between groups $(p=0.2)$. Three days of VitD administration significantly increased the serum IL-10 level in VitD group, just before operation $(5.3 \pm 3.8$ vs. $1 \pm 0 \mathrm{ng} / \mathrm{mL}$, $p=0.001)$. There was also an increase in serum IL-10 level in both groups with a constant difference after the operation ( $370 \pm 65$ vs. $180 \pm 93 \mathrm{ng} / \mathrm{mL}, p<0.001)$. In the first postoperative day, the serum IL-10 level has been sharply decreased with a persevered difference $(8.7 \pm 6.2$ vs. $5.4 \pm 4.8 \mathrm{ng} / \mathrm{mL}, p=0.02$ ) (Fig. 2).

By sorting information of patients according to AKI occurrence, there was no difference in demographic and operative data between patients. However, the AKI patients had more mediastinal bleeding $(p=0.02)$, received more blood transfusion during operation $(p=0.05)$ and postoperative in the ICU $(p=0.04)$, and need more intraaortic balloon pump $(p=0.01)$. In AKI patients, there was a significant increase in urine IL-18, KIM-1 level just after operation, and the next day ( $p<0.001$ for both), compared to non-AKI patients. Finally, according to AKI occurrence, there was no difference in the serum level of IL-10 between patients ( $p=0.8)$.

\section{Discussion}

In the present study, the effect of short-term high-dose VitD supplementation on renal function was examined by measuring the $\mathrm{Cr}$ and tubular injury markers in patients with VitD deficiency undergoing coronary surgery. In this clinical trial, VitD failed to decrease the frequency of postoperative AKI. However, VitD administration was associated with a significantly elevated serum level of the anti-inflammatory IL-10 both before and after the surgery.

VitD plays a key role in the immunomodulatory function for attenuating the production of proinflammatory cytokines and increasing anti-inflammatory markers $[8,9,11,13,19]$. VitD is found to be renoprotective in angiotensin II-induced renal injury in the experimental model through inhibition of mitochondrial dysfunction, inflammatory process, and attenuating renal cell apoptosis $[3,14]$. In a clinical setting, ischemic reperfusion injury during $\mathrm{CPB}$ can lead to mitochondrial destruction, renal cell injury, and even cell death [3]. The increased level of inflammatory cytokines is associated with AKI development [3]. VitD protects the tubular cells against the recruitment of inflammatory cells in experimental models [12]. In the mouse model, VitD pretreatment ( $24 \mathrm{~h}$ before ischemia) attenuates renal ischemic reperfusion injury (tubular necrosis and apoptotic cell death) by decreasing the inflammatory cell infiltration and inflammatory cytokines [20, 21]. This property has been confirmed in human proximal tubular cell experiments using different pathways [1517]. In patients with renal failure, VitD administration decreased the serum level of inflammatory cytokines and urinary biomarker [13]. 


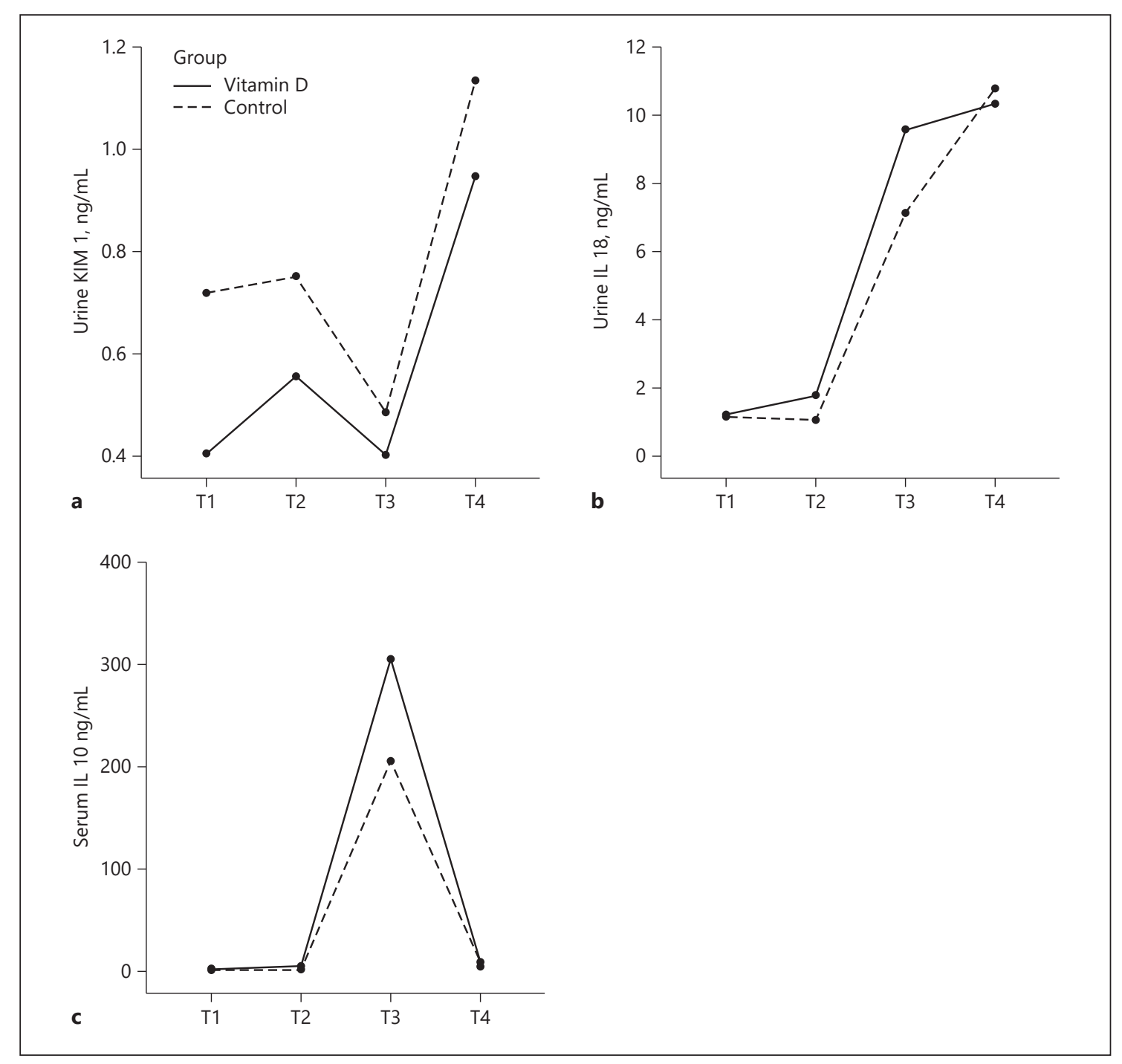

Fig. 2. The urinary level of kidney biomarkers over time. KIM-1 (a), IL-18 (b), and serum level of IL-10 (c). There were significant time effects for KIM-1 and IL-18, with no statistical difference between the groups. VitD administration significantly increased the serum level of IL-10 before and after surgery. Time points: (T1) before intervention, (T2) before anesthesia induction, (T3) at the end of operation, and (T4) the first day after operation. Data are expressed as mean \pm SD. Solid line: vitamin D group $(n=50)$, dashed line: control group $(n=61)$. VitD, vitamin D.

The key mechanisms of CSA-AKI are a hemodynamic disturbance, inflammation, and nephrotoxic agents [1, 3]. These factors operate in different ways with interconnection pathways contributing to tubular epithelial cell damage and endothelial cell dysfunction, which overlap and crosstalk each other $[1,3,21]$. Also, inflammatory factors could affect both vascular endothelial and tubular epithelial cell integrity. There is a correlation between the extent and duration of AKI and tubular markers [1].
There is no effective and verified treatment for established AKI, therefore, every attempt for manipulating the modifiable risk factors (both patient-related and operative-related) of AKI to improve clinical outcome is appreciated [1]. Despite the experimental studies, which have proven the renoprotective property of $\mathrm{VitD}$, in this study, there was no protecting effect against AKI in VitD group. It is possible that, although VitD improves anti-inflammatory state before and after cardiac surgery, due to the 
interaction and involvement with other factors, it could not exhibit its important effect to make a significant difference. This gap may also be due to the probably different mechanism between inducing a direct renal ischemic reperfusion in experimental studies and renal involvement during $\mathrm{CPB}$.

\section{Limitation}

This is a single-center experience in the small sample size of coronary patients who were at low risk for AKI occurrence. Furthermore, adding a higher risk population (especially patients with heart failure, either systolic or diastolic failure or CKD patients) might have enhanced the clinical effect of VitD for nephroprotection in the future studies. It cannot be concluded whether the increase in serum level of IL-10 is related to improved outcome. Although previous studies have proven this association, it was out of our design study.

\section{Conclusion}

This prospective study with a relative homogenous patient population suggests that VitD may be able to control inflammation in ischemic conditions by increasing antiinflammatory biomarkers. Further investigation is required to determine its clinical significance and whether an optimal VitD status participates in renal protection during cardiac surgery.

\section{Acknowledgement}

The authors thank Professor Dabbagh for all his valuable help.

\section{Statement of Ethics}

Written informed consent was obtained from each study participant before enrollment in the study. This study protocol was approved by the Ethics Committee of the Shahid Beheshti University of Medical Sciences (Approval number IR.SBMU.MSP. REC.1397.633).

\section{Conflict of Interest Statement}

The authors have no conflicts of interest to disclose.

\section{Funding Sources}

The present study was supported by grants from the Research Department of the School of Medicine, Shahid Beheshti University of Medical Sciences, Tehran, Iran (no. 13732). This article has been extracted from the thesis written by Miss. Eslami (Registration No. 456).

\section{Author Contributions}

M.F. and P.E.: study design; M.F., M.H., M.B., R.B., and S.M.M.: data collection; M.F. and F.P.: data analysis; S.A.Z., F.P., and R.B.: data interpretation; D.I., M.F., P.E., M.B., and M.H.: literature search and generation of figures; M.F. and P.E.: writing of the manuscript. All authors gave their final approval of the submitted version.

\section{References}

1 Ortega-Loubon C, Fernández-Molina $\mathrm{M}$, Carrascal-Hinojal Y, Fulquet-Carreras E. Cardiac surgery-associated acute kidney injury. Ann Card Anaesth. 2016;19(4):687.

2 Scrascia G, Guida P, Rotunno C, de Luca Tupputi Schinosa L, Paparella D. Anti-inflammatory strategies to reduce acute kidney injury in cardiac surgery patients: a metaanalysis of randomized controlled trials. Artif Organs. 2014;38(2):101-12.

3 Wang Y, Bellomo R. Cardiac surgery-associated acute kidney injury: risk factors, pathophysiology and treatment. Nat Rev Nephrol. 2017;13(11):697.

4 Malek M, Nematbakhsh M. Renal ischemia/ reperfusion injury; from pathophysiology to treatment. J Renal Inj Prev. 2015;4(2):20.
5 Small DM, Coombes JS, Bennett N, Johnson DW, Gobe GC. Oxidative stress, anti-oxidant therapies and chronic kidney disease. $\mathrm{Ne}$ phrology. 2012;17(4):311-21.

6 Belletti A, Licheri M, Bove T. Perioperative renal pharmacological protection during cardiovascular surgery. Visceral vessels and aortic repair. Springer; 2019. p. 177-94.

7 Foroughi M, Argani H, Hassntash SA, Hekmat M, Majidi M, Beheshti M, et al. Lack of renal protection of ultrafiltration during cardiac surgery: a randomized clinical trial. J Cardiovasc Surg. 2014;55(3):407-13.

8 Lee TW, Lee TI, Chang CJ, Lien GS, Kao YH, Chao TF, et al. Potential of vitamin $\mathrm{D}$ in treating diabetic cardiomyopathy. Nutr Res. 2015; 35(4):269-79.
9 Dozio E, Briganti S, Vianello E, Dogliotti G, Barassi A, Malavazos AE, et al. Epicardial adipose tissue inflammation is related to vitamin $\mathrm{D}$ deficiency in patients affected by coronary artery disease. Nutr Metab Cardiovasc Dis. 2015;25(3):267-73.

10 Arnson Y, Itzhaky D, Mosseri M, Barak V, Tzur B, Agmon-Levin N, et al. Vitamin D inflammatory cytokines and coronary events: a comprehensive review. Clin Rev Allergy Immunol. 2013;45(2):236-47.

11 Pludowski P, Holick MF, Grant WB, Konstantynowicz J, Mascarenhas MR, Haq A, et al. Vitamin D supplementation guidelines. J Steroid Biochem Mol Biol. 2018;175:125-35.

12 Tan X, Wen X, Liu Y. Paricalcitol inhibits renal inflammation by promoting vitamin $\mathrm{D}$ receptor-mediated sequestration of NF-kappaB signaling. J Am Soc Nephrol. 2008;19(9):1741-52.
Vitamin D and Renal Inflammatory

Response during Cardiac Surgery
Cardiorenal Med 2021;11:52-58

DOI: $10.1159 / 000511058$ 
13 Lucisano S, Arena A, Stassi G, Iannello D, Montalto G, Romeo A, et al. Role of paricalcitol in modulating the immune response in patients with renal disease. Int J Endocrinol. 2015;2015:765364.

14 Shen Q, Bi X, Ling L, Ding W. 1,25-Dihydroxyvitamin D3 attenuates angiotensin IIinduced renal injury by inhibiting mitochondrial dysfunction and autophagy. Cell Physiol Biochem. 2018;51(4):1751-62.

15 Hong YA, Yang KJ, Jung SY, Park KC, Choi $\mathrm{H}$, Oh JM, et al. Paricalcitol pretreatment attenuates renal ischemia-reperfusion injury via prostaglandin E2 receptor EP4 pathway. Oxi Med Cell Longev. 2017;2017:5031926.
16 Park JS, Choi HI, Bae EH, Ma SK, Kim SW. Paricalcitol attenuates indoxyl sulfate-induced apoptosis through the inhibition of MAPK, Akt, and NF-kB activation in HK-2 cells. Korean J Intern Med. 2019;34(1):146.

17 Hong YA, Yang KJ, Jung SY, Chang YK, Park CW, Yang CW, et al. Paricalcitol attenuates lipopolysaccharide-induced inflammation and apoptosis in proximal tubular cells through the prostaglandin E2 receptor EP4. Kidney Res Clin Pract. 2017;36(2):145.

18 Payani N, Foroughi M, Dabbagh A. The effect of intravenous administration of active recombinant factor VII on postoperative bleeding in cardiac valve reoperations; a randomized clinical trial. Anesth Pain Med. 2015; 5(1):e22846.
19 Assimon MM, Salenger PV, El-Fawal HA, Mason DL. Nutritional vitamin D supplementation in haemodialysis: a potential vascular benefit? Nephrology. 2012;17(3):23742.

20 Hwang HS, Yang KJ, Park KC, Choi HS, Kim $\mathrm{SH}$, Hong SY, et al. Pretreatment with paricalcitol attenuates inflammation in ischemia-reperfusion injury via the up-regulation of cyclooxygenase-2 and prostaglandin E2. Nephrol Dial Transplant. 2012;28(5):1156-66.

21 Lee JW, Kim SC, Ko YS, Lee HY, Cho E, Kim $\mathrm{MG}$, et al. Renoprotective effect of paricalcitol via a modulation of the TLR4-NF- $\kappa B$ pathway in ischemia/reperfusion-induced acute kidney injury. Biochem Biophys Res Commun. 2014;444(2):121-7. 